\title{
OPEN Efficient radiational outcoupling of electromagnetic energy from hyperbolic metamaterial resonators
}

\author{
Ildar Yusupov ${ }^{1,5 \bowtie}$, Dmitry Filonov ${ }^{2,5}$, Tatyana Vosheva², Viktor Podolskiy ${ }^{3} \&$ \\ Pavel Ginzburg ${ }^{2,4,5}$
}

Hyperbolic metamaterials were initially proposed in optics to boost radiation efficiencies of quantum emitters. Adopting this concept for antenna design allows approaching long-standing contests in radio physics. For example, broadband impedance matching, accompanied with moderately high antenna gain, is among the existent challenges. Here we propose employing hyperbolic metamaterials for a broadband impedance matching, while a structured layer on top of a metamaterials slab ensures an efficient and directive energy outcoupling to a free space. In particular, a subwavelength loop antenna, placed underneath the matching layer, efficiently excites bulk metamaterial modes, which have well-resolved spatial-temporal separation owing to the hypebolicity of effective permeability tensor. Interplaying chromatic and modal dispersions enable to map different frequencies into non overlapping spatial locations within a compact subwavelength hyperbolic slab. The outcoupling of energy to the free space is obtained by patterning the slab with additional resonant elements, e.g. high index dielectric spheres. As the result, two-order of magnitude improvement in linear gain of the device is predicted. The proposed new architecture can find a use in applications, where multiband or broadband compact devices are required.

Electromagnetic materials play an important role in tailoring and controlling electromagnetic energy flow. While electrodynamic equations are scalable in respect to an operation frequency, material properties are subject to a considerable chromatic dispersion ${ }^{1}$. As a result, architectures of passive electromagnetic devices for optical and centimeter wave ranges are conceptually different. For example, metal components are very attractive for waveguide and antenna design at $\mathrm{GHz}$ range because of their almost vanishing losses. For example, copper demonstrates properties of a perfect electric conductor (PEC) within a good approximation. On the other hand, metals are quite lossy at the optical domain and are rarely used in applications where long-range communication is needed. Quite a few efforts have been done in order to control electromagnetic properties of materials by applying different techniques. In this context, the approach of metamaterials allows tailoring permittivities and permeabilities almost on demand by accurate subwavelength structuring of periodic unit cells ${ }^{2-4}$. Hyperbolic metamaterials were found to be quite attractive in optical domain, as they suggest achieving relatively high local density of electromagnetic states (LDOS), as it was proposed theoretically ${ }^{5}$ and then demonstrated experimentally, e.g. ${ }^{6,7}$. The key property, which is responsible for achieving a range of peculiar effects, is an extremely high anisotropy of a susceptibility tensor (ether electric or magnetic). Quite a few realizations of hyperbolic metamaterials have been demonstrated in the optical regime, where free standing metal rods ${ }^{8-10}$, metal-dielectric layers ${ }^{11-14}$ or graphene stack ${ }^{15,16}$ are the most widely studied. The physical effect in the beforehand mentioned designs is based on shaping negative permittivity components (e.g. silver, gold, or doped semiconductors), which support surface or localized plasmon resonances ${ }^{17,18}$. Replicating characteristics of hyperbolic metamaterials from optical to $\mathrm{GHz}$ domains is quite challenging since negative susceptibility (either $\varepsilon$ or $\mu$ ) materials are rather rare in nature. Nevertheless, arrays or loaded wires or split ring resonators were designed and demonstrated sufficient negative values of susceptibility tensors ${ }^{15,16,19-21}$. The drawback of this approach an inherently narrowband

${ }^{1}$ Department of Physics and Engineering, ITMO University, Saint Petersburg 197101, Russia. ${ }^{2}$ Center of Photonics and 2D materials, Moscow Institute of Physics and Technology, Dolgoprudny 141700, Russia. ${ }^{3}$ Department of Physics and Applied Physics, University of Massachusetts Lowell, One University Avenue, Lowell, MA 01854, USA. ${ }^{4}$ School of Electrical Engineering, Tel Aviv University, 69978 Tel Aviv, Israel. ${ }^{5}$ These authors contributed equally: IldarYusupov, Dmitry Filonov and Pavel Ginzburg ${ }^{\bowtie}$ email: ildar.yusupov@metalab.ifmo.ru 


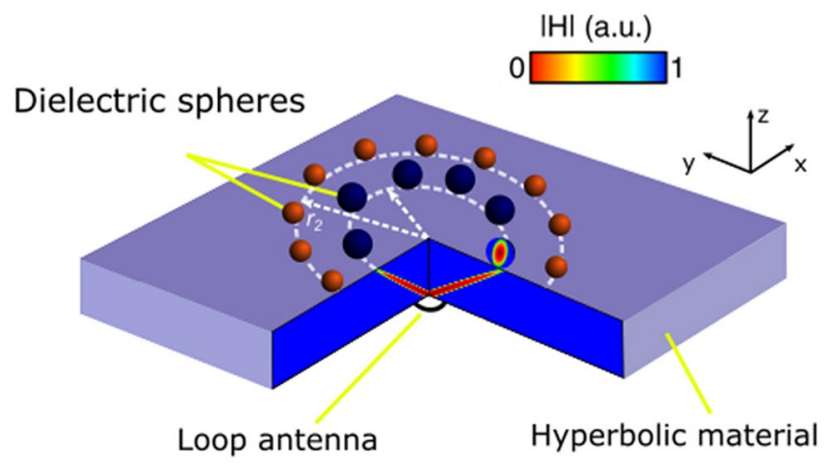

Figure 1. Structure's layout-hyperbolic metamaterial substrate with circular array of high-index dielectric particles on top. Those arrays are responsible to provide a broadband outcoupling of energy. Each array corresponds to a specific frequency band. The structure is excited by a nonresonant loop antenna, placed underneath the slab.

operation, which is the direct consequence of the resonance-based methodology. In order to achieve a broadband hyperbolicity at the $\mathrm{GHz}$ range, a different design has been proposed. In particular, an array of corrugated wires, supporting spoof plasmon waves, was shown to demonstrate hyperbolic properties in a potentially broader spectral window ${ }^{22}$. The possibility to replicate broadband hyperbolic properties into centimeter waves domain suggest considering this material as a component in antenna design.

Material degrees of freedom are rarely considered as tuning parameters in conservative antenna design approaches. In a vast majority of cases, a geometric optimization over a set of shapes is used to achieve desired characteristics, dictated by an application. Metamaterial approach allows introducing additional design flexibility as compared with conventional approaches. For example, high-gain antenna design ${ }^{23}$, scattering suppression devices $^{24,25}$, resonators for radiation efficiency enhancement ${ }^{26}$, hyperbolic metamaterial-based antennae ${ }^{27,28}$, and several others configurations have been proposed.

An inherent drawback of using electromagnetic materials in antenna applications is the leakage of radiation onto high-index substrates. Similar challenges are faced in solar-cell devices, where light harvesting and photon recycling into semiconductor layers are required. Quite a few different approaches have been developed and include introducing surface roughness, patterned surfaces, arrays of particles (harvesting optical antennas), and others ${ }^{29}$. Light harvesting and extraction from hyperbolic metamaterials also attracted an attention, since standard techniques, described above, can fail. The main reason is high LDOS inside a hyperbolic substrate, which result in a highly directional scattering into the bulk ${ }^{30}$. In this case, bulk modes are the preferential scattering channel, and free-space propagating modes are not excited efficiently.

The goal of our investigation on pathways to develop a new generation of antenna devices with metamaterialbased components is to demonstrate a strategy of electromagnetic energy outcoupling from hyperbolic substrates, in some sense bringing the advances of optical science to $\mathrm{GHz}^{31}$. Our approach is based on incorporating arrays of resonant scatterers on top of a hyperbolic metamaterial layer (Fig. 1). In this case, an interplay between interaction channels is tailored to maximize the scattering to the free space modes. We have found an optimal balance and have demonstrated more than 100-fold enhancement of extraction efficiency, which can be obtained in several frequency bands, depending on application requirements.

The report is organized as follows: the relevance of hyperbolic metamaterial to antennae design is discussed first and then followed by a set of optimizations of energy extracting elements. The last part is designated for antenna gain optimization and radiation pattern analysis, which comes before a conclusion.

\section{Results}

An infinite metamaterial slab with an effective diagonal permeability (Eq. 1) has been considered as a test object.

$$
\mu_{r}=\left(\begin{array}{ccc}
1 & 0 & 0 \\
0 & 1 & 0 \\
0 & 0 & \mu_{z z}(\omega)
\end{array}\right) .
$$

Similar susceptibility was demonstrated experimentally in Ref. ${ }^{21}$, where arrays of near-field coupled split rings resemble an artificial material with negative effective permeability in a resonance vicinity. Here, a Drude-type of dispersion for the nontrivial tensor component was assumed (Eq. 2) and it is depicted in Fig. 2 a.

$$
\mu_{z z}(\omega)=\mu_{\infty}-\frac{\omega_{p}^{2}}{\omega\left(\omega-i \omega_{c}\right)},
$$

where $\mu_{\infty}=1, \omega_{p}=25 \bullet 10^{9} \frac{\mathrm{rad}}{\mathrm{s}}, \omega_{c}=12 \bullet 10^{8} \frac{\mathrm{rad}}{\mathrm{s}}$.

A radiating element (a small $2 \mathrm{~mm}$ radius loop, connected to a feeding coaxial cable) was brought to a close proximity of the slab. The dimensions of the slab are $200 \times 200 \times 20 \mathrm{~mm}$. It is worth noting that the radiation efficiency of a deeply subwavelength loop is inherently small. While it can be significantly improved by matching 
(a) Dispersion model

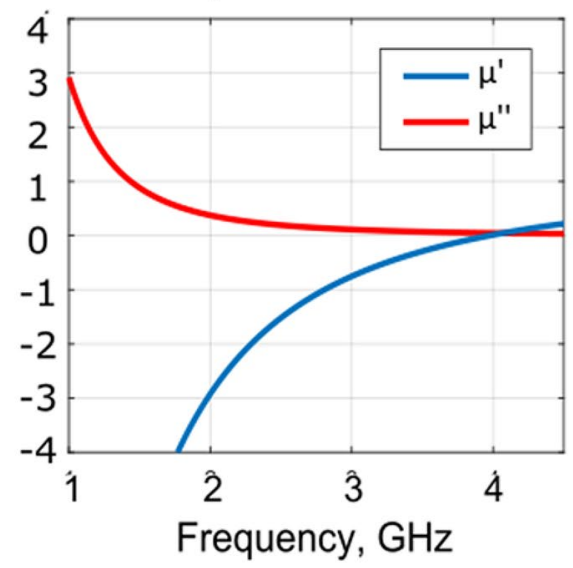

(b)

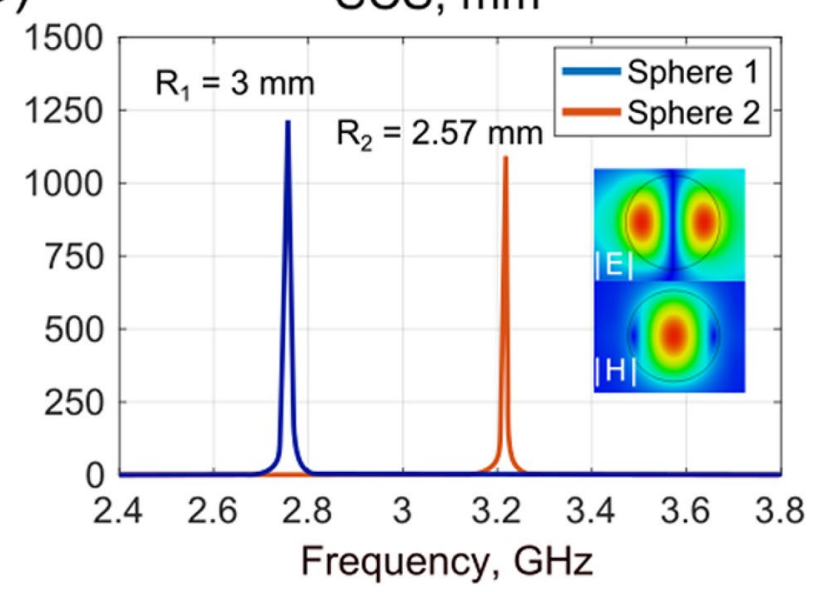

Figure 2. (a) Dispersion of a nontrivial permittivity tensor component (Eq. 1). Real and imaginary parts are blue and red lines correspondingly. (b) Scattering cross sections of high-index dielectric spheres (radiuses are $3 \mathrm{~mm}$ and $2.57 \mathrm{~mm}, \varepsilon=330$ ). $|\mathrm{E}|$ and $|\mathrm{H}|$ field colormaps (arbitrary units) at a magnetic dipole resonance of spheres are in the inset.
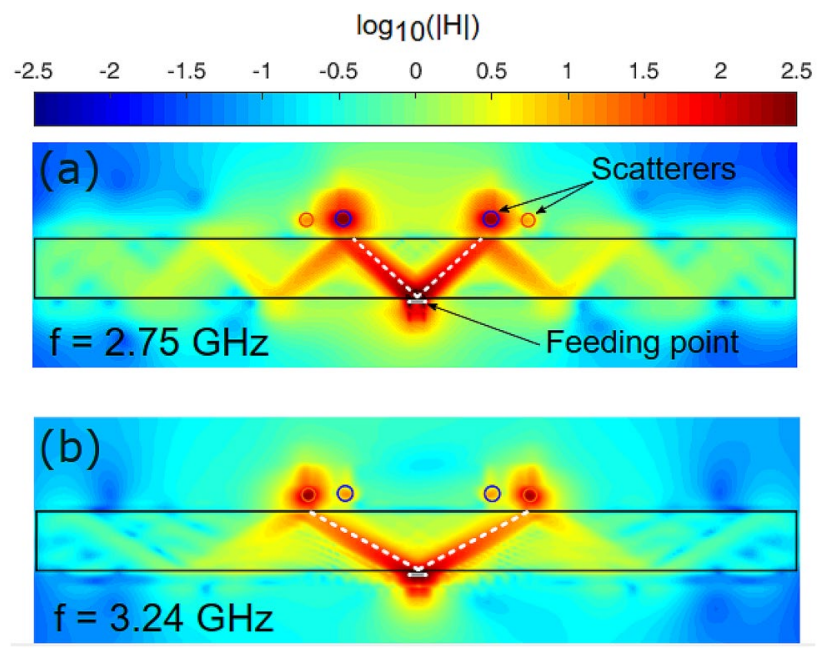

Figure 3. Electromagnetic energy extraction from hyperbolic metamaterial slab. Logarithmic color maps (arbitrary units) of the magnetic field amplitude $(|\mathrm{H}|)$ at two different frequencies of the excitation (a) $2.75 \mathrm{GHz}$. (b) $3.24 \mathrm{GHz}$ ).

circuits, sufficiently broadband and impedance-matched radiation cannot be obtained without violating ChuHarrington limit ${ }^{32}$ and involving non-Foster matching circuits ${ }^{33}$. Nevertheless, putting an impedance mismatched loop next to a hyperbolic metamaterial results in an efficient excitation of extraordinary modes inside the hyperbolic structures. An unusual (in comparison to free space scenarios) radiation pattern, known as resonance cones $^{34}$, can be seen in Fig. 3, is a hallmark of hyperbolic metamaterials (e.g. Refs. ${ }^{35,36}$ ). A three-dimensional (3D) emission pattern within the slab is a cone with an apex, situated close to the radiating element. The cone hits the upper facet of the slab and re-traced back into the structure. If the inherent ohmic losses of the hyperbolic metamaterial are moderately low, the cone is reflected from the upper surface and additional inverted one is created. Figure 3 illustrates such multiple reflection scenario, which will be suppressed in the case of moderately high losses. The latter scenario is almost always the case for the optical domain hyperbolic metamaterials (e.g. Ref. ${ }^{8}$ ).

The opening angle of the radiation cone inside the metamaterial has strong chromatic dispersion. This aspect will be the key for demonstrating a dual band energy extraction with resonant elements. The angle of the radiation cone can be estimated as a ratio between ordinary and extra-ordinary tensor components:

$$
\tan ^{2} \theta(\omega)=\left|\frac{\mu_{t}(\omega)}{\mu_{z z}(\omega)}\right|
$$

where $\mu_{t} \equiv \mu_{x x}=\mu_{y y}$ is the tangential component of the permeability tensor. Figure 3 shows the near-field magnetic field distribution for 2 frequencies, demonstrating the chromatic dispersion of the opening angle. The 
similar effect enables hyper-spectroscopy at optical frequencies ${ }^{37}$. This property will allow obtaining operation at multiple frequency bands and control each channel separately without a need in complex multiplexing circuitry, as it will be shown hereinafter.

After achieving an efficient launching of energy into the hyperbolic substrate, the next step is to out couple the electromagnetic radiation to the free space ${ }^{38}$. If no additional effort is performed, the upper facet almost completely reflects the energy, leaving an exponentially decaying field in the free space above the surface. In order to solve this extraction problem, an efficient electromagnetic scatterer should perturb this exponentially decaying field and convert it to propagate in the free space. Several parameters should be considered here in order to obtain an efficient design. In particular, a scatterer's position in respect to the surface should be optimized to improve an overall radiation efficiency. For example, if the scatterer is placed too far from the surface, the exponentially-decaying field will not excite it and the reflection back to the substrate will predominate the free space scattering channel. On the other hand, if the scatterer is in the very close proximity to the surface, the rescattered radiation will leak again into the substrate and not to the free space. Hence, it is reasonable to assume that an engineering tradeoff does exist.

In order to perform the energy extraction optimization, the following setup has been chosen. High-index dielectric spheres were considered as resonating elements. Those structures accommodate moderately high-quality factors along with relatively low internal losses. Those elements can be realized as e.g. silicone nanospheres for optical domain applications (e.g. Refs. ${ }^{39,40}$ ) and as ceramic resonators for $\mathrm{GHz}$ waves (e.g. Ref. ${ }^{41}$ ). In the latter case, loss tangents can be as small as $\left.10^{-4}\right)$. High index particles possess a rich family of Mie modes. Here we employ the first magnetic dipolar resonance. Scattering cross-section of a standalone sphere $\left(\mathrm{R}_{1}=3 \mathrm{~mm}, \mathrm{R}_{2}=2.57 \mathrm{~mm}\right.$, $\varepsilon=330$ ) appears in Fig. $2 \mathrm{~b}$ and a well-pronounced resonance can be seen. The field distribution of the resonant particle is given in the inset, indicating it's moderately high localization. Dimensions of the particles were calculated in the way they resonate at the desired frequencies, chosen for the dual band operation.

The next step is to optimize the distance between the sphere and the metamaterial's surface. This will be the major parameter, affecting the scattering efficiency to the upper half-space. The out coupling process strongly depends on two key parameters, which within a good approximation, can be considered as if they operate sequentially. The first one is the excitation efficiency. A small polarizable sphere is excited by the evanescent field, leaking from the metamaterial substrate (Fig. 4a). The dipole moment, which is developed on the particle, is proportional to the sphere's polarizability and the local magnetic field amplitude. Hence, placing the particle far from the boundary will significantly reduce its excitation efficiency. The next step, after the sphere has developed a dipole moment on it, is the scattering. Efficiency of this process can be estimated by placing a small radiating dipole next to the metamaterial boundary. This scenario has been considered analytically in a series of works, related to optical domain applications, e.g. Refs. ${ }^{30,42}$. Main results can be retrieved numerically in a quite straightforward fashion. Figure $4 \mathrm{~b}$ shows the radiation efficiency (vector Poynting flux through the upper hemi-sphere). Putting a radiating dipole in a close proximity to the hyperbolic substrate results in a strong quenching - the main power flows into the slab, since it supports high LDOS, preferential to scattering. Typical dependency of this quenching is $1 / l^{3}$, where $l$ is the distance to the upper facet. Enlarging the distance causes more efficient radiation to the free space. This factor saturates when there is no near field coupling between the radiating dipole and the slab. The efficiency of the entire process can be estimated as a product of the two factors depicted on panels a and $b$, indicating the existence of the optimal scatterer's position.

Figure $4 \mathrm{c}$ shows the normalized total radiated power (TRP) for the entire scenario. Here 2 circular arrays of equidistant spheres ( 8 and 12 in inner and outer array, respectively) were considered and the same excitation scheme, used for calculating results on Fig. 3, is employed. The TRP is normalized to the scenario, where the upper facet of the metamaterial is flat and no spheres are present. The ratio demonstrates the overall improvement of energy out coupling. As it was expected, the TRP has an optimum for a certain separation distance, which also depends on the operating frequency. This chromatic dispersion is expected since both field decay and particle's polarizability are frequency dependent. In overall, the optimal distance for both cases is between 3 and $5 \mathrm{~mm}$.

The last stage is the assessment of the dual-band performance of the device. For this purpose we calculated the normalized gain of the structure as a function of frequency. An additional important issue in antenna applications is side lobes, partially affected by the radiation from edges.

Furthermore, front to back ratio also a subject for maximization. A standard technique in antenna design is to enclose a device (apart from its aperture) within a metal shield. Here we place a perfect electric conductor (PEC) on the side and the bottom facets of the structure, leaving the upper plane uncovered. The excitation loop is placed directly within the enclosure. Figure 5 compares performance of the device with and without arrays of spheres and illustrates the frequency dependence of the normalized antenna gain along with far-field diagrams. Several main features can be clearly seen. The first one is two distinct peaks around the frequencies, which were chosen for the dual band operation. The second aspect is a relatively high gain improvement -2 orders of magnitude with respect to the unpatterned structure. This result should not be confused with an efficient extraction of energy from high-index layers and does not violate theoretical limits ${ }^{43}$. Furthermore, in order to approach capabilities of a high-performance device, internal material losses should be further reduced. It is also worth noting that the array factor, multiplied by the gain of the single element does not provide a single radiation beam. This results from the fact that a single sphere is predominantly polarized perpendicular to the surface and, consequently, it does not radiate perpendicular to it. While this might be an issue in standard antenna applications, the obtained radiation pattern can find a use in radar tracking (e.g. monopulse) applications and several others. 
(a)

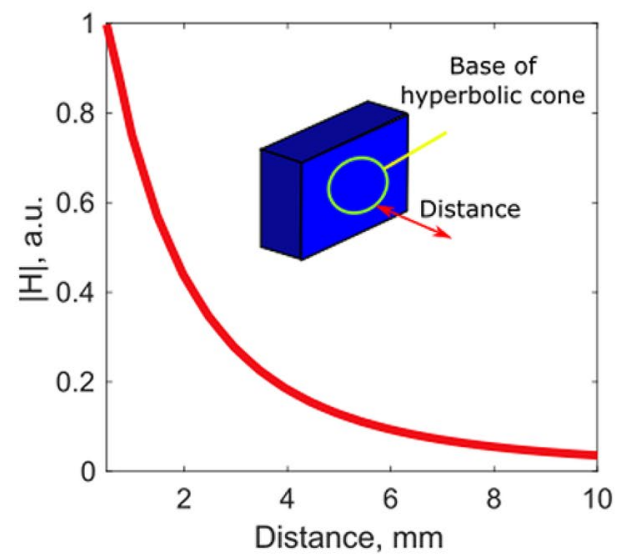

(b)

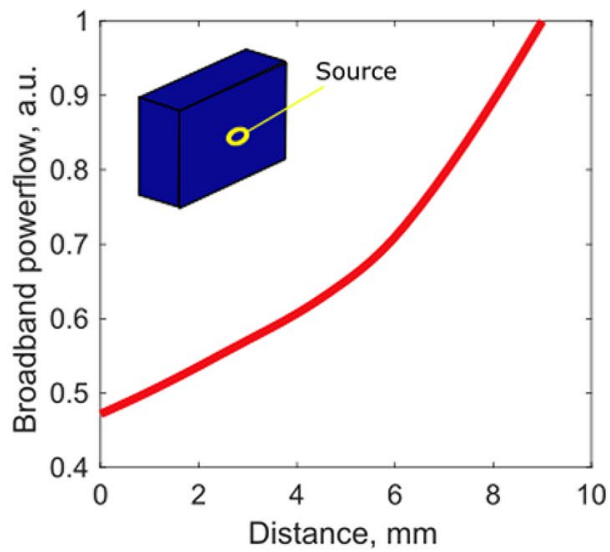

(c)

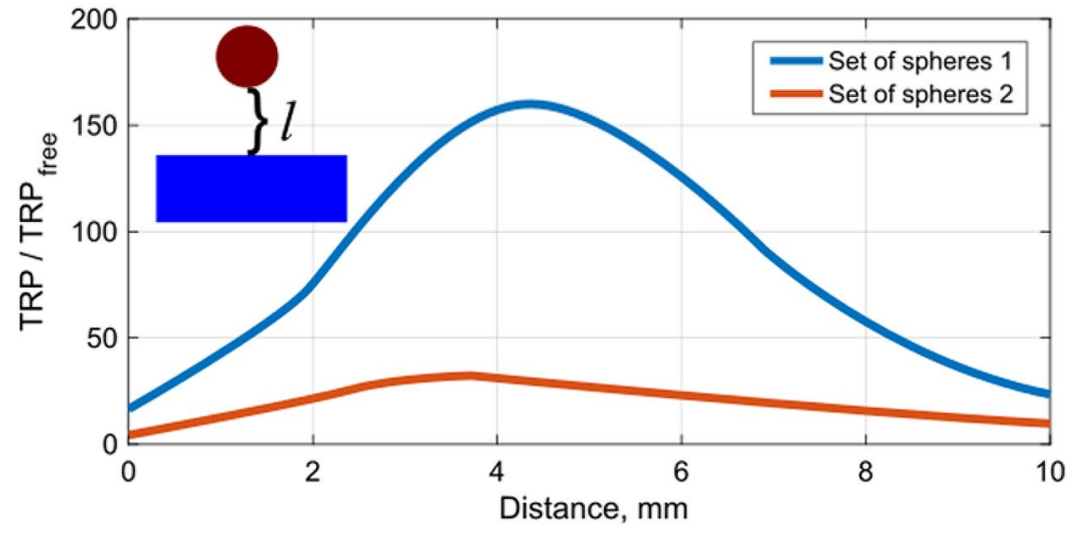

Figure 4. (a) Normalized magnetic field amplitude, as a function of the distance from the metamaterial surface. The excitation scheme is similar to Fig. 3a. (b) Power to the upper hemisphere, as a function of a dipolar emitter's distance from the metamaterial surface (the radiating dipole is polarized perpendicular to the surface). (c) Total radiated power (TRP) to the upper hemisphere, when the entre scenario of Fig. 3 is considered (circular array of spheres on top of the metamaterial slab). Blue and red curves correspond to the two set of spheres, designed to provide the dual band operation. The presented values are normalized to the total radiation efficiency of the device with a flat facet (without any spheres present).

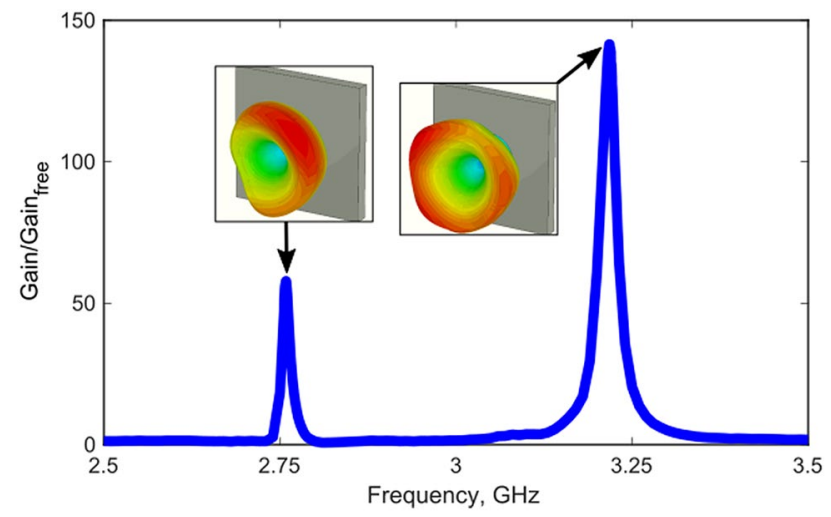

Figure 5. Normalized gain spectra for the pair of chosen frequencies. The normalization is the ratio of gain, obtained for the device with circular array of spheres and without it. Insets-far-field radiation patterns. 


\section{Conclusion}

It was shown that hyperbolic metamaterials can contribute to the endeavor of demonstrating compact broadband antenna devices. While a broadband matching and directivity are rather hard to accommodate together and achieve with standard design approaches, the concept of broadband impedance matching, adopted from the optical domain can break this commonly accepted engineering trade off. Here we demonstrated a design of a dual-band antenna device, which is based on 2 key elements-hyperbolic metamaterial slab for a broadband impedance matching and circular arrays of resonat elements for an efficient out coupling. The optimized structure shows more than 2 orders of magnitude improvement of the antenna gain (linear scale) and paves a way for further improvement of other antenna characteristics. The key step for demonstrating the proposed concept in antenna applications is a metamaterial realization with moderately low internal losses and miniature unit cell. The later factor is essential to ensure applicability of the effective medium model. Furthermore, the unit cell granularity should be finer than the size of the resonating elements, used for the radiation outcoupling. For example, realizations, demonstrated in Ref. ${ }^{21}$ can be further miniaturized with meandering techniques, enhanced by lumped elements, plugged in relevant parts of resonating elements.

Received: 10 August 2020; Accepted: 2 December 2020

Published online: 14 December 2020

\section{References}

1. John, D. J. Classical Electrodynamics 3rd edn. (Wiley, Hoboken, 1998).

2. Cai, W. \& Shalaev, V. Optical Metamaterials-Fundamentals and Applications. (Springer, New York, 2010). https://doi. org/10.1007/978-1-4419-1151-3.

3. Engheta, N. \& Ziolkowski, R. W. Metamaterials: Physics and Engineering Explorations. Metamaterials: Physics and Engineering Explorations, 1st edn (Wiley-IEEE Press, Hoboken, 2006). https://doi.org/10.1002/0471784192.

4. Capolino, F. Applications of Metamaterials (CRC Press, Boca Raton, 2009).

5. Jacob, Z., Smolyaninov, I. I. \& Narimanov, E. E. Broadband Purcell effect: Radiative decay engineering with metamaterials. Appl. Phys. Lett. 100, 181105 (2012).

6. Tumkur, T. et al. Control of spontaneous emission in a volume of functionalized hyperbolic metamaterial. Appl. Phys. Lett. 99, 151115 (2011).

7. Ginzburg, P. et al. Spontaneous emission in non-local materials. Light Sci. Appl. 6, e16273 (2017)

8. Ginzburg, P. et al. Manipulating polarization of light with ultrathin epsilon-near-zero metamaterials. Opt. Express 21, 14907 (2013).

9. Kabashin, A. V. et al. Plasmonic nanorod metamaterials for biosensing. Nat. Mater. 8, 867-871 (2009).

10. Wangberg, R., Elser, J., Narimanov, E. E. \& Podolskiy, V. A. Nonmagnetic nanocomposites for optical and infrared negativerefractive-index media. J. Opt. Soc. Am. B 23, 498 (2006).

11. Krishnamoorthy, H. N. S., Jacob, Z., Narimanov, E., Kretzschmar, I. \& Menon, V. M. Topological transitions in metamaterials. Science 336, 205-209 (2012).

12. Zhukovsky, S. V. et al. Experimental demonstration of effective medium approximation breakdown in deeply subwavelength alldielectric multilayers. Phys. Rev. Lett. 115, 177402 (2015).

13. Podolskiy, V. A. \& Narimanov, E. E. Strongly anisotropic waveguide as a nonmagnetic left-handed system. Phys. Rev. B Condens. Matter Mater. Phys. 71, 201101 (2005).

14. Hoffman, A. J. et al. Negative refraction in semiconductor metamaterials. Nat. Mater. 6, 946-950 (2007).

15. Iorsh, I. V., Mukhin, I. S., Shadrivov, I. V., Belov, P. A. \& Kivshar, Y. S. Hyperbolic metamaterials based on multilayer graphene structures. Phys. Rev. B Condens. Matter Mater. Phys. 87, 075416 (2013).

16. Chang, Y. C. et al. Realization of mid-infrared graphene hyperbolic metamaterials. Nat. Commun. 7, 1-7 (2016).

17. Novotny, L. \& Hecht, B. Principles of Nano-Optics. Principles of Nano-Optics Vol. 9781107005 (Cambridge University Press, Cambridge, 2012).

18. Berkovitch, N., Ginzburg, P. \& Orenstein, M. Nano-plasmonic antennas in the near infrared regime. J. Phys. Condens. Matter 24, $073202(2012)$.

19. Pendry, J. B., Schurig, D. \& Smith, D. R. Controlling electromagnetic fields. Science 312, 1780-1782 (2006).

20. Shelby, R. A., Smith, D. R. \& Schultz, S. Experimental verification of a negative index of refraction. Science 292, 77-79 (2001).

21. Filonov, D., Shmidt, A., Boag, A. \& Ginzburg, P. Artificial localized magnon resonances in subwavelength meta-particles. Appl. Phys. Lett. 113, 123505 (2018).

22. Fan, B., Filonov, D., Ginzburg, P. \& Podolskiy, V. A. Low-frequency nonlocal and hyperbolic modes in corrugated wire metamaterials. Opt. Express 26, 17541 (2018).

23. Zhao, Y. Design of high-gain, wideband antenna using microwave hyperbolic metasurface. AIP Adv. 6, 055022 (2016).

24. Shalin, A. S. et al. Scattering suppression from arbitrary objects in spatially dispersive layered metamaterials. Phys. Rev. B Condens. Matter Mater. Phys. 91, 125426 (2015).

25. Filonov, D. S., Shalin, A. S., Iorsh, I., Belov, P. A. \& Ginzburg, P. Controlling electromagnetic scattering with wire metamaterial resonators. J. Opt. Soc. Am. A 33, 1910 (2016).

26. Slobozhanyuk, A. P. et al. Purcell effect in hyperbolic metamaterial resonators. Phys. Rev. B Condens. Matter Mater. Phys. 92,195127 (2015).

27. Valagiannopoulos, C. A., Mirmoosa, M. S., Nefedov, I. S., Tretyakov, S. A. \& Simovski, C. R. Hyperbolic-metamaterial antennas for broadband enhancement of dipole emission to free space. J. Appl. Phys. 116, 163106 (2014).

28. Inam, F. A., Ahmed, N., Steel, M. J. \& Castelletto, S. Hyperbolic metamaterial resonator-antenna scheme for large, broadband emission enhancement and single-photon collection. J. Opt. Soc. Am. B 35, 2153 (2018).

29. Nayak, P. K., Mahesh, S., Snaith, H. J. \& Cahen, D. Photovoltaic solar cell technologies: analysing the state of the art. Nat. Rev. Mater. 4, 269-285 (2019)

30. Kidwai, O., Zhukovsky, S. V. \& Sipe, J. E. Effective-medium approach to planar multilayer hyperbolic metamaterials: Strengths and limitations. Phys. Rev. B 053842, 1-12 (2012).

31. Galfsky, T., Gu, J., Narimanov, E. E. \& Menon, V. M. Photonic hypercrystals for control of light-matter interactions. Proc. Natl. Acad. Sci. U. S. A. 114, 5125-5139 (2017).

32. Harrington, R. F. Time-Harmonic Electromagnetic Fields (Wiley-IEEE Press, Hoboken, 2001).

33. Stearns, S. D. Non-foster circuits and stability theory. In IEEE Antennas and Propagation Society, AP-S International Symposium (Digest) 1942-1945 (2011). https://doi.org/10.1109/APS.2011.5996883.

34. Balmain, K. G., Lüttgen, A. A. E., Kremer, P. C. \& Rogers, E. S. Resonance cone formation, reflection, refraction, and focusing in a planar anisotropic metamaterial. IEEE Antennas Wirel. Propag. Lett. 1, 146-149 (2002). 
35. Kapitanova, P. V. et al. Photonic spin Hall effect in hyperbolic metamaterials for polarization-controlled routing of subwavelength modes. Nat. Commun. 5, 3226 (2014).

36. Ginzburg, P. et al. Self-induced torque in hyperbolic metamaterials. Phys. Rev. Lett. 111, 036804 (2013).

37. Narimanov, E. Hyperstructured illumination. ACS Photon. 3, 1090-1094 (2016).

38. Sun, G., Khurgin, J. B. \& Soref, R. A. Plasmonic light-emission enhancement with isolated metal nanoparticles and their coupled arrays. J. Opt. Soc. Am. B 25, 1748 (2008).

39. Kuznetsov, A. I., Miroshnichenko, A. E., Fu, Y. H., Zhang, J. \& Luk'yanchuk, B. Magnetic light. Sci. Rep. 2, 492 (2012).

40. Markovich, D. et al. Enhancement of artificial magnetism via resonant bianisotropy. Sci. Rep. 6, 22546 (2016).

41. Baryshnikova, K. et al. Giant magnetoelectric field separation via anapole-type states in high-index dielectric structures. Phys. Rev. B 98, 165419 (2018)

42. Ivinskaya, A. et al. Optomechanical manipulation with hyperbolic metasurfaces. ACS Photon. 5, 4371-4377 (2018).

43. Roberts, C. M., Cook, T. A. \& Podolskiy, V. A. Metasurface-enhanced transparency. J. Opt. Soc. Am. B 34, D42 (2017).

\section{Acknowledgements}

This work was supported by the Russian Science Foundation under Project № 20-19-00480). Pavel Ginzburg acknowledges partial support by Binational Science Foundation (project 2016059) and PAZY Foundation.

\section{Author contributions}

All authors reviewed the manuscript.

\section{Competing interests}

The authors declare no competing interests.

\section{Additional information}

Correspondence and requests for materials should be addressed to I.Y.

Reprints and permissions information is available at www.nature.com/reprints.

Publisher's note Springer Nature remains neutral with regard to jurisdictional claims in published maps and institutional affiliations.

(c) (i) Open Access This article is licensed under a Creative Commons Attribution 4.0 International License, which permits use, sharing, adaptation, distribution and reproduction in any medium or format, as long as you give appropriate credit to the original author(s) and the source, provide a link to the Creative Commons licence, and indicate if changes were made. The images or other third party material in this article are included in the article's Creative Commons licence, unless indicated otherwise in a credit line to the material. If material is not included in the article's Creative Commons licence and your intended use is not permitted by statutory regulation or exceeds the permitted use, you will need to obtain permission directly from the copyright holder. To view a copy of this licence, visit http://creativecommons.org/licenses/by/4.0/.

(C) The Author(s) 2020 